\title{
Metformin promotes cell proliferation and osteogenesis under high glucose condition by regulating the ROS-AKT-mTOR axis
}

\author{
RENYI ZHOU ${ }^{1}$, YUE MA ${ }^{2}$, SHUI QIU ${ }^{1}$, ZUNLEI GONG ${ }^{1}$ and XIAOSHU ZHOU ${ }^{1}$ \\ ${ }^{1}$ Department of Orthopedics, The First Affiliated Hospital of China Medical University; ${ }^{2}$ Department of Respiratory and \\ Critical Care Medicine, Shengjing Hospital of China Medical University, Shenyang, Liaoning 110001, P.R. China
}

Received March 16, 2020; Accepted June 4, 2020

DOI: $10.3892 / \mathrm{mmr} .2020 .11391$

\begin{abstract}
Metformin, a cost-effective and safe orally administered antidiabetic drug used by millions of patients, has exhibited great interest for its potential osteogenic-promoting properties in different types of cells, including mesenchymal stem cells (MSCs). Diabetic osteopathy is a common comorbidity of diabetes mellitus; however, the underlying molecular mechanisms of metformin on the physiological processes of MSCs, under high glucose condition, remain unknown. To determine the effects of metformin on the regulatory roles of proliferation and differentiation in MSCs, under high glucose conditions, osteogenesis after metformin treatment was detected with Alizarin Red S and ALP staining. The results demonstrated that high glucose levels significantly inhibited cell proliferation and osteogenic differentiation under high glucose conditions. Notably, addition of metformin reversed the inhibitory effects induced by high glucose levels on cell proliferation and osteogenesis. Furthermore, high glucose levels significantly decreased mitochondrial membrane potential (MMP), whereas treatment with metformin helped maintain MMP. Further analysis of mitochondrial function revealed that metformin significantly promoted ATP synthesis, mitochondrial DNA mass and mitochondrial transcriptional activity, which were inhibited by high glucose culture. Furthermore, metformin significantly scavenged reactive oxygen species (ROS) induced by high glucose levels, and regulated the ROS-AKT-mTOR axis inhibited by high glucose levels, suggesting the protective effects of metformin against high glucose levels via regulation of the ROS-AKT-mTOR axis. Taken together, the results of the present study demonstrated the protective role of metformin on the physiological processes of MSCs, under high glucose
\end{abstract}

Correspondence to: Professor Xiaoshu Zhou, Department of Orthopedics, The First Affiliated Hospital of China Medical University, 155 Nanjing North Street, Shenyang, Liaoning 110001, P.R. China

E-mail: 13889291613@163.com

Key words: metformin, reactive oxygen species, cell proliferation, osteogenesis, mitochondrial membrane potential condition and highlighted the potential molecular mechanism underlying the effect of metformin in promoting cell proliferation and osteogenesis under high glucose condition.

\section{Introduction}

Diabetes is considered a chronic metabolic disease worldwide, which is characterized by high blood glucose levels due to inadequate insulin secretion or/and activity (1). Worldwide, diabetes presented in $3.82 \%$ of the general population in 2017 (2). Diabetes is associated with different types of disease due to durable hyperglycemia, such as periodontal disease, microvascular disease, nephropathy, retinopathy, neuropathy and osteopathy $(3,4)$. Diabetic osteopathy is one of the most common types of disease associated with diabetes, which often results in skeletal fragility, osteoporosis and bone pain $(5,6)$. Diabetics are reported to have an osteopathy rate of $0.1-6.8 \%$ (7-9). Its poor prognosis decreases the quality of life of patients and negatively affects the social economy (1). However, the development of effective novel therapeutic strategies for diabetic osteopathy remains limited as its pathogenic mechanism is not yet fully understood.

Metformin is a relatively cheap and efficient antihyperglycemic biguanide, which is extensively used as a first-line treatment for type 2 diabetes mellitus (T2dm) (10). In addition to its predominant role in lowering high blood glucose levels, metformin also exerts an osteogenic effect by promoting the differentiation of preosteoblasts and mesenchymal stem cells (MSCs) (11,12). Furthermore, metformin has been demonstrated to exhibit protective effects against injury induced by high glucose on osteoblastic cells, including proliferation (13). Smith et al (9) reported that metformin induces osteoblastic differentiation of human induced pluripotent stem cell-derived MSCs by activating LKB1/AMP-activated protein kinase signaling. Notably, metformin has also been demonstrated to affect osteoblasts against the effect of high glucose levels on proliferation, suggesting that metformin is directly involved in the regulation of both osteogenic cells and osteoblasts (14).

Hyperglycemic damage is frequently caused by long-term exposure to high glucose levels (15). During long-term exposure, high glucose levels induce generation and accumulation of reactive oxygen species (ROS), which is closely associated with the pathogenesis of diabetic osteopathy (16). Zhen et al (13) reported that ROS induced 
by high glucose levels regulate ROS-AKT (protein kinase B)-mTOR signaling that is downstream of ROS, and tightly regulate high glucose-associated proliferation and apoptosis. Metformin was also demonstrated to decrease ROS accumulation and promote osteogenesis, potentially by regulating the ROS-AKT-mTOR axis (17). However, whether metformin is involved in regulating the ROS-AKT-mTOR axis in MSCs, under high glucose conditions still remains unclear.

The present study aimed to investigate the effect of metformin on the regulatory roles of proliferation and differentiation in MSCs, under high glucose conditions. To achieve this, Alizarin Red S staining was performed to detect osteogenesis of MSCs following metformin treatment, and hallmarkers of osteogenesis, including ALP, osteocalcin, OPN and run-related transcription factor 2, were detected. The results of the present study provided novel insight into the regulatory effects of metformin on MSCs, and provided a better understanding on the protective role metformin plays against high glucose-induced damage and a novel therapeutic strategy to avid from suffering from diabetic osteopathy.

\section{Materials and methods}

Animal information and cell culture. A total of two female C57B mice (4-weeks old, 20-25 g in weight, bought from Chengdu Dashuo Experimental Animal Limited Company) were maintained under SPF conditions. The mice were housed at room temperature with $12 \mathrm{~h}$ light/dark cycles, 50-65\% humidity, and access to standard chow and water. All animal experiments were approved by the Ethics Committee of The First Affiliated Hospital of China Medical University (approval no. 2019014).

After one week mice were euthanatized by $30 \mathrm{mg} / \mathrm{kg}$ sodium pentobarbital and cervical dislocation to collect the femur. The bone marrow was subsequently washed out using $5 \mathrm{ml}$ ice cold DMEM-F12 (Thermo Fisher Scientific, Inc.) and centrifuged at $400 \mathrm{x} \mathrm{g}$ for $10 \mathrm{~min}$ at $4^{\circ} \mathrm{C}$. Then, bone marrow was treated using SoniConvert ${ }^{\circledR}$ sonicator (DocSense) to obtain single cells. The cells were resuspended in $5 \mathrm{ml}$ ice cold DMEM-F12 and $3 \mathrm{ml}$ ice cold Ficoll solution (Jixing Biotech Inc.), and centrifuged at $1,000 \times \mathrm{g}$ for $30 \mathrm{~min}$ at $4^{\circ} \mathrm{C}$. The white-fog layer on the surface of the Ficoll was collected and equal volume of DMEM-F12 ( 500 $\mu \mathrm{l})$ was added followed by centrifugation at $400 \mathrm{xg}$ at $4^{\circ} \mathrm{C}$ for $20 \mathrm{~min}$. The pellet was collected and incubated in DMEM-F12 supplemented with $10 \%$ FBS and $1 \%$ mix of penicillin-streptomycin (Thermo Scientific, Inc.) for the primary culture at $37^{\circ} \mathrm{C}$ for $\geq 4$ weeks. DMEM-F12 supplemented with $10 \%$ FBS and $1 \%$ mix of penicillin-streptomycin was used as the growth medium for cell proliferation analysis, while differentiating medium (cat. no. MUXMT-90021; Cyagen Biosciences, Inc.) was used for cell differentiation analysis.

CCK-8 assay cell viability was determined by the CCK-8 assay. Briefly, cells $\left(5 \times 10^{4}\right)$ were cultured in 96-well plates. Then, $10 \mu \mathrm{l}$ of freshly prepared CCK- 8 solution was added to the cell culture for 2 -h co-incubation. The absorbance was measured at $620 \mathrm{~nm}$ by using a microplate reader (Synergy 2 Multi-Mode Microplate Reader; BioTek Instruments,
Inc.). The cell viability was expressed as the optical density (OD) 620 .

Alkaline phosphatase (ALP) assay. Bone marrow stromal cells (BMSCs) were seeded into 6-well plates at a density of $1 \times 105$ cells/well and maintained in growth medium and differentiating medium for 3, 5, 14 and 21 days, respectively. Cells were washed three times with ice-cold PBS, digested with $0.25 \%$ trypsin and suspended. Pelleted cells were resuspended in extraction lysis buffer containing $50 \mathrm{mM}$ Tris- $\mathrm{HCl} 8.0$, $150 \mathrm{mM} \mathrm{NaCl}, 0.5 \mathrm{mM}$ EDTA, $0.25 \%$ Nonidet P(NP)-40 and incubated for $30 \mathrm{~min}$ at $4^{\circ} \mathrm{C}$. The supernatant was collected and total protein concentration was measured using a bicinchoninic acid assay (Pierce; Thermo Fisher Scientific, Inc.). The results were analyzed at a wavelength of $520 \mathrm{~nm}$ using a Synergy 2 Multi-Mode microplate reader (BioTek Instruments, Inc.) and the ALP activity assay detection kit (cat. no. A509-1; Nanjing Jiancheng Bioengineering Institute). Osteogenic differentiation was determined by measuring the area stained for ALP, using MetaMorph Imaging software (version 7.7.8; Universal Imaging, Inc.). All experiments were performed in triplicate.

Alizarin Red $S$ (ARS) staining assay. BMSCs $\left(1 \times 10^{5}\right)$ were cultured in growth medium for 14 and 21 days, respectively. Cells were subsequently washed three times with ice-cold PBS and fixed with $4 \%$ formaldehyde in PBS for $10 \mathrm{~min}$ at room temperature. Following a brief wash with PBS, cells were stained with ARS solution (0.5\%, Sigma-Aldrich; Merck $\mathrm{KGaA}$ ) supplemented with a final concentration of $40 \mathrm{mM}$ ARS ( $\mathrm{pH} 4.2$ ) for $30 \mathrm{~min}$ at room temperature. Subsequently, cells were washed five times with PBS to inhibit non-specific staining and the stained cells were imaged under an X71 (U-RFL-T) fluorescence microscope (Olympus Corporation). All experiments were performed in triplicate.

Reverse transcription-quantitative $(R T-q) P C R$. BMSCs cells $\left(1 \times 10^{6}\right)$ were cultured in differentiating medium for 24 and $48 \mathrm{~h}$, respectively. Total RNA was extracted using TRIzol reagent (Thermo Fisher Scientific, Inc.), according to the manufacturer's protocol and RNA concentration was measured using a UV spectrophotometer (Thermo Fisher Scientific, Inc.). cDNA was synthesized from $1 \mu \mathrm{g}$ of total RNA using the Reverse Transcriptase kit (cat. no. QP056; Guangzhou RiboBio Co., Ltd.) at $37^{\circ} \mathrm{C}$ for $40 \mathrm{~min}$ and inactivated at $85^{\circ} \mathrm{C}$ for 10 min. qPCR was subsequently performed using the SYBR-Green Master Mix (Thermo Fisher Scientific, Inc.), in a ABI7500 system (Applied Biosystems; Thermo Fisher Scientific, Inc.). The following primer sequences were used for qPCR: ALP forward, 5'-CCTTGAAAAATGCCCTGAAA-3' and reverse, 5'-CTTGGAGAGAGCCACAAAGG-3'; osteocalcin (OCN) forward, 5'-GAGGGCAGTAAGGTGGTGAA-3' and reverse, 5'-CCTAAACGGTGGTGCCATAG-3'; OPN forward, 5'-AGTGTGAGGAAGGGCGTTAC-3' and reverse, 5'-AATGTGCTGCAGTTCGTGTG-3'; runt-related transcription factor 2 (RUNX2) forward, 5'-AAATGCCTCCGCTGTT ATGAA-3' and reverse, 5'-GCTCCGGCCCACAAATCT-3'; COX I forward, 5'-GGAGCAGTATTCGCCATCAT-3' and reverse, 5'-CGACGAGGTATCCCTGCTAA-3'; COX 3 forward, 5'-GAACATACCAAGGCCACCAC-3' and reverse, 5'-TAATTCCTGTTGGGGGTCAG-3'; ND1 forward, 
5'-CTCCCTATTCGGAGCCCTAC-3' and reverse, 5'-GGA GCTCGATTTGTTTCTGC-3'; Cyb forward, 5'-GTCGGC GAAGAAAAATGTGT-3' and reverse, 5'-AAGCTGCTC ACAGAGGGGTA-3'; and $\beta$-actin forward, 5'-CATGTACGT TGCTATCCAGGC-3' and reverse, 5'-CTCCTTAAT GTCACGCACGAT-3'. The following thermocycling conditions were used for qPCR: Initial denaturation $98^{\circ} \mathrm{C} 5 \mathrm{~min}, 35$ of cycles of denaturation at $98^{\circ} \mathrm{C}$ for $10 \mathrm{sec}$, annealing and elongation at $60^{\circ} \mathrm{C}$ for $1 \mathrm{~min}$; and final extension at $60{ }^{\circ} \mathrm{C}$ for $10 \mathrm{~min}$.

To detect mitochondrial DNA contents, total DNA was isolated using Genome DNA isolation kit (DocSense) with $20 \mathrm{ng}$ of DNA being used as a template. The following thermocycling conditions were used for qPCR: Initial denaturation $98^{\circ} \mathrm{C} 5 \mathrm{~min}, 35$ of cycles of denaturation at $98^{\circ} \mathrm{C}$ for $10 \mathrm{sec}$, annealing and elongation at $60^{\circ} \mathrm{C}$ for $1 \mathrm{~min}$. The specific primers used were followed: $12 \mathrm{~S}$ rDNA forward: 5'-ACCGCGGTCATACGATTAAC-3'; reverse: 5'-AGTACCGCCAAGTCCTTTGA-3'; 18S rDNA forward: 5'-TCAATCTCGGGTGGCTGAACG-3'; reverse: 5'-GGACCAGAGCGAAAGCATTTG-3'. All PCR data were quantified using the $2^{-\triangle \Delta C q}$ method (18).

Western blotting. BMSCs were cultured in differentiating medium for $48 \mathrm{~h}$. Cells $\left(1 \times 10^{6}\right)$ suspended in $0.5 \mathrm{ml}$ of Laemmle buffer (50 mM tris pH6.8, 1.25\% SDS, $10 \%$ glycerol) were lysed using SoniConvert sonicator (DocSense) and denatured by heating at $100^{\circ} \mathrm{C}$ for $15 \mathrm{~min}$. Then, protein concentration was determined using BCA kit (Sigma-Aldrich; Merck $\mathrm{KGaA})$. Total protein $(20 \mu \mathrm{g})$ was separated in each lane via 6-12\% gradient SDS-PAGE electrophoresis, transferred onto nitrocellulose membranes (EMD Millipore) and subsequently blocked with PBS containing 5\% skimmed milk (Beyotime Institute of Biotechnology) at room temperature for $30 \mathrm{~min}$. The membranes were incubated with primary antibodies against: AKT (1:3,000; cat. no. ab9905), AKT phosphor T308 (1:2,000; cat. no. ab38449), mTOR (1:1,000; cat. no. ab2732), mTOR phosphor S2448 (1:1,000; cat. no. ab109268) and $\beta$-actin (1:5,000; cat. no. ab8226; all from Abcam) for $1 \mathrm{~h}$ at room temperature. Membranes were washed three times with PBS containing 0.1\% Tween-20 (Beyotime Institute of Biotechnology) and subsequently incubated with horseradish peroxidase-labeled goat anti-rabbit secondary antibody (1:5,000; cat. no. ab7090; Abcam) for $1 \mathrm{~h}$ at room temperature. Proteins bands were visualized on X-ray film using enhanced chemiluminescence substrate (Thermo Fisher Scientific, Inc.) and quantified using ImageJ software (version-2.0; National Institutes of Health).

Cell cycle analysis. BMSCs were cultured in growth medium for $48 \mathrm{~h}$. Cells $\left(1 \times 10^{6}\right)$ were suspended and fixed with ice-cold $75 \%$ ethyl alcohol (1 ml; Sigma-Aldrich; Merck KGaA), and stored overnight at $4^{\circ} \mathrm{C}$. Cells were subsequently collected and washed twice with ice-cold PBS, prior to incubation with $100 \mu \mathrm{l}$ RNase A and $400 \mu \mathrm{l}$ propidium iodide (PI; both from Sigma-Aldrich; Merck KGaA) for $30 \mathrm{~min}$ at room temperature in the dark. Following an additional incubation for $30 \mathrm{~min}$ at $4^{\circ} \mathrm{C}$, cell cycle distribution was assessed using a FACS LSRII flow cytometer (BD Biosciences). All experiments were performed in triplicate.
ROS detection. BMSCs were cultured in differentiating medium for $48 \mathrm{~h}$. To scavenge accumulated ROS, $10 \mu \mathrm{M}$ of antioxidant NAC was added for 2-h incubation. The addition of the same volume of DMSO was considered as the control (mock) group. Cells $\left(1 \times 10^{5}\right)$ were co-cultured with $10 \mu \mathrm{M}$ 2',7'-dichlorodihydrofluorescein diacetate (DCFH-DA; Beyotime Institute of Biotechnology) for $30 \mathrm{~min}$ at $37^{\circ} \mathrm{C}$ in the dark. Cells were washed three times with PBS and fluorescence was detected via fluorescence microscopy (magnification, x100). Fluorescence was measured at wavelengths of $488 \mathrm{~nm}$ excitation and $525 \mathrm{~nm}$ emission using a two-laser Navios flow cytometer (Beckman Coulter, Inc.). All experiments were performed in triplicate.

$J C-1$ staining. BMSCs were cultured in differentiating medium for $48 \mathrm{~h}$. In order to measure mitochondrial membrane potential (MMP), cells $\left(1 \times 10^{5}\right)$ were stained with JC-1 (cat. no. T3168; Thermo Fisher Scientific, Inc.), according to the manufacturer's protocol. Briefly, cells were washed twice with ice-cold PBS and stained with JC-1 work solution for $30 \mathrm{~min}$ at $37^{\circ} \mathrm{C}$ in the dark. Following incubation, the supernatant was discarded and cells were re-washed twice with PBS, prior to imaging via fluorescence microscopy (X71; Olympus Corporation; magnification, x100). All experiments were performed in triplicate.

ATP measurement. BMSCs were cultured in differentiating medium for $48 \mathrm{~h}$. In order to assess ATP synthesis, cells $\left(5 \times 10^{5}\right)$ were stained with reagents from the ATP Bioluminescent Assay kit (cat. no. FLAA-1KT; Sigma-Aldrich; Merck KGaA) at room temperature for $20 \mathrm{~min}$, and subsequently maintained in solution containing $0.22 \mathrm{M}$ sucrose, $0.12 \mathrm{M}$ mannitol, $40 \mathrm{mM}$ Tricine, pH 7.5 and $1 \mathrm{mM}$ EDTA (all from Sigma-Aldrich; Merck $\mathrm{KGaA}$ ) at room temperature for $10 \mathrm{~min}$, and the supernatant was analyzed using an Optocomp I luminometer (MGM Instruments, Inc.). Grounded mitochondrial lysate was incubated in buffer containing $5 \mathrm{mM} \mathrm{MgCl}_{2}, 10 \mathrm{mM} \mathrm{KH}_{2} \mathrm{PO}_{4}$, $0.2 \%$ Bovine serum albumin, $0.1 \mathrm{mM}$ ADP and $54 \mu \mathrm{M}$ APP at $4^{\circ} \mathrm{C}$ for $10 \mathrm{~min}$. Glutamate and malate (both $10 \mathrm{mM}$ ) were used as the substrates. All experiments were performed in triplicate.

Statistical analysis. All data were presented in mean \pm standard deviation. Statistical analysis was performed using GraphPad Prism software (GraphPad Software, Inc.; version 5.01). Unpaired Student's t-test was used to determine differences between two independent groups, while one- or two-way analysis of variance (ANOVA) was used compare differences between multiple groups. Bonferroni's post hoc test was performed for all pairwise comparisons following ANOVA. All experiments were performed in triplicate. $\mathrm{P}<0.05$ (2-tailed) was considered to indicate a statistically significant difference.

\section{Results}

High glucose inhibits osteogenic differentiation of BMSCs in differentiating medium. In order to assess the effect of high, medium and regular glucose conditions on the osteogenesis of BMSCs in growth medium and differentiating medium, respectively, ALP staining was performed following cell culture for 3 and 5 days. Under differentiating conditions, ALP staining significantly decreased under high and medium 
A

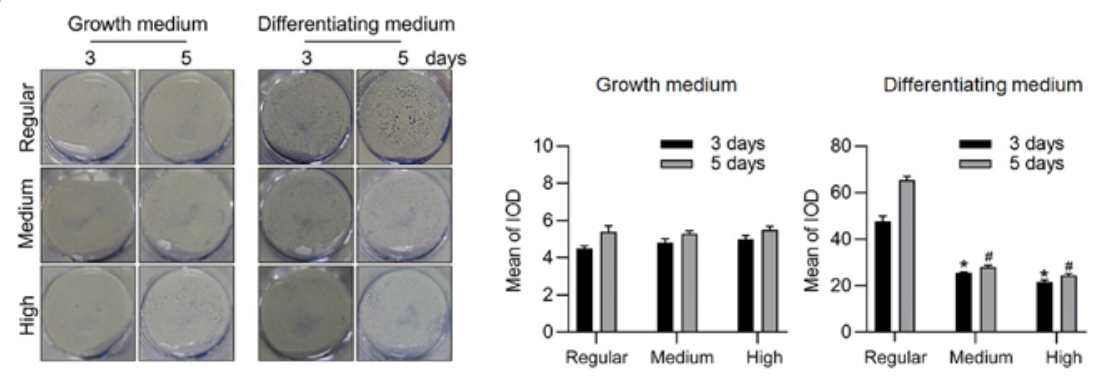

B
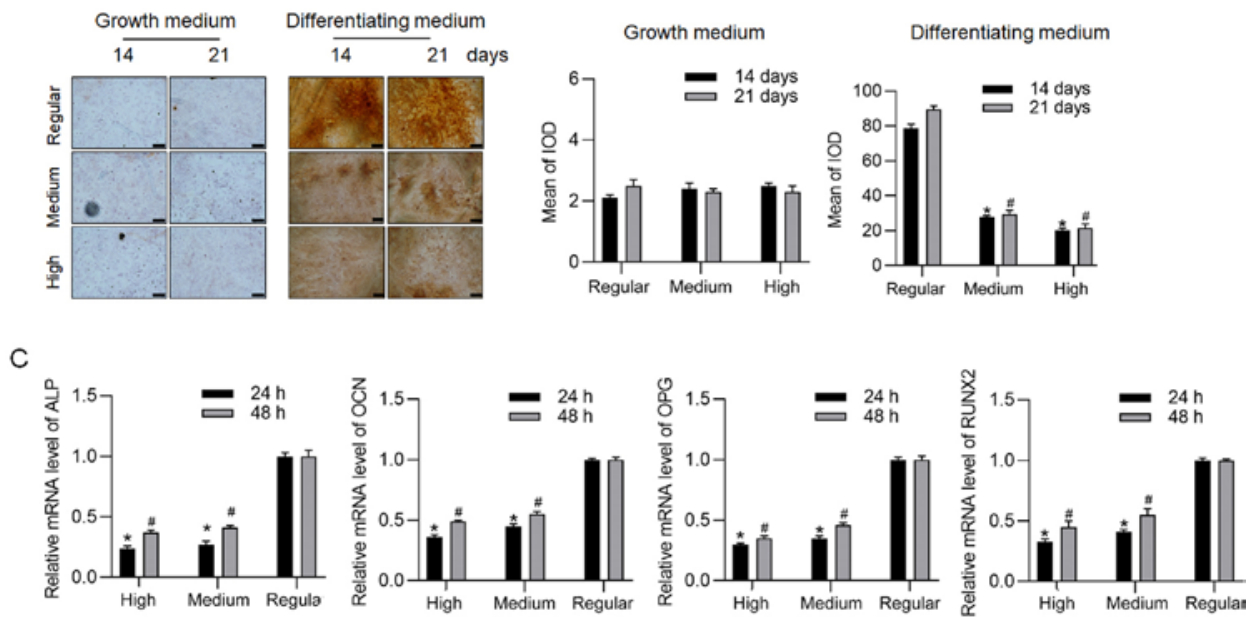

Figure 1. Effects of high, medium and regular glucose conditions on osteogenic differentiation of BMSCs. (A) ALP and (B) Alizarin Red S staining assays were performed following incubation for 3 and 5 days (magnification, $x 40$; scale bar $=50 \mu \mathrm{m}$ ). (C) mRNA expression levels of ALP, OCN, OPG and RUNX2, of BMSCs cultured in differentiating medium were detected via reverse transcription-quantitative PCR analysis. " $\mathrm{P}<0.05$ vs. regular group at 3 days; ${ }^{*} \mathrm{P}<0.05$ vs. regular group at 5 days. BMSCs, bone marrow stromal cells; ALP, alkaline phosphatase; OCN, osteocalcin; OPG, osteoprotegerin; RUNX2, runt-related transcription factor 2; IOD, integrated optical density.

glucose conditions at both 3 and 5 days (Fig. 1A). Similarly, under differentiating medium for 14 and 21 days, ARS staining significantly decreased under both high and medium glucose conditions (Fig. 1B). In order to confirm osteogenesis, markers of osteogenesis, including ALP, OCN, osteoprotegerin (OPG) and RUNX2 were detected via RT-qPCR analysis following cell culture in differentiating medium for 24 and $48 \mathrm{~h}$, which were notably induced at the early stage of differentiation, but not the later stage of differentiation. As expected, high and medium glucose conditions significantly decreased the mRNA levels of these genes (Fig. 1C). Based on the dose-dependent effect of glucose, the high glucose condition was selected for further experimentation.

Metformin regulates cell proliferation and differentiation under specific culture conditions. The present study set out to determine whether metformin exerts different roles under different culturing conditions. Cell viability was assessed in BMSCs cultured in growth medium supplemented with regular or high glucose at days 1-5. Although no significant differences were observed between days 1-5, high glucose condition significantly decreased cell viability at day 5 compared with the regular glucose group, which was reversed following addition of metformin (Fig. 2A).

Cell cycle distribution was subsequently assessed in cells treated at days 3 and 5 via flow cytometric analysis. The results demonstrated that addition of metformin significantly decreased the proportion of cells in the $G_{1} / G_{0}$ phase at both days 3 and 5 compared with the high glucose group, suggesting the promoting effect of metformin on cell proliferation (Fig. 2B).

The effect of metformin on osteogenesis of BMSCs cultured in differentiating medium, under regular or high glucose conditions was assessed. Consistent with previous findings (13), the results of the present study demonstrated that high glucose condition inhibited osteogenesis of BMSCs cultured in differentiating medium for 14 days, the effect of which was significantly reversed following the addition of metformin (Fig. 3A and B). Addition of metformin significantly increased the mRNA levels of hallmarkers of osteogenesis, including ALP, OCN, OPG and RUNX2 (Fig. 3C). Taken together, these results suggest that metformin is remarkably involved in regulating both proliferation and osteogenesis of BMSCs, which are inhibited under high glucose condition.

Metformin promotes mitochondrial maintenance and function inhibited by high glucose condition. The effects of metformin on high glucose condition in differentiating medium was subsequently assessed. Following incubation in differentiating medium for $48 \mathrm{~h}$ at $37^{\circ} \mathrm{C}$, cells were stained with JC-1, and aggregates and monomers were observed. The results demonstrated that high glucose condition significantly decreased aggregates and increased monomers, both of which 

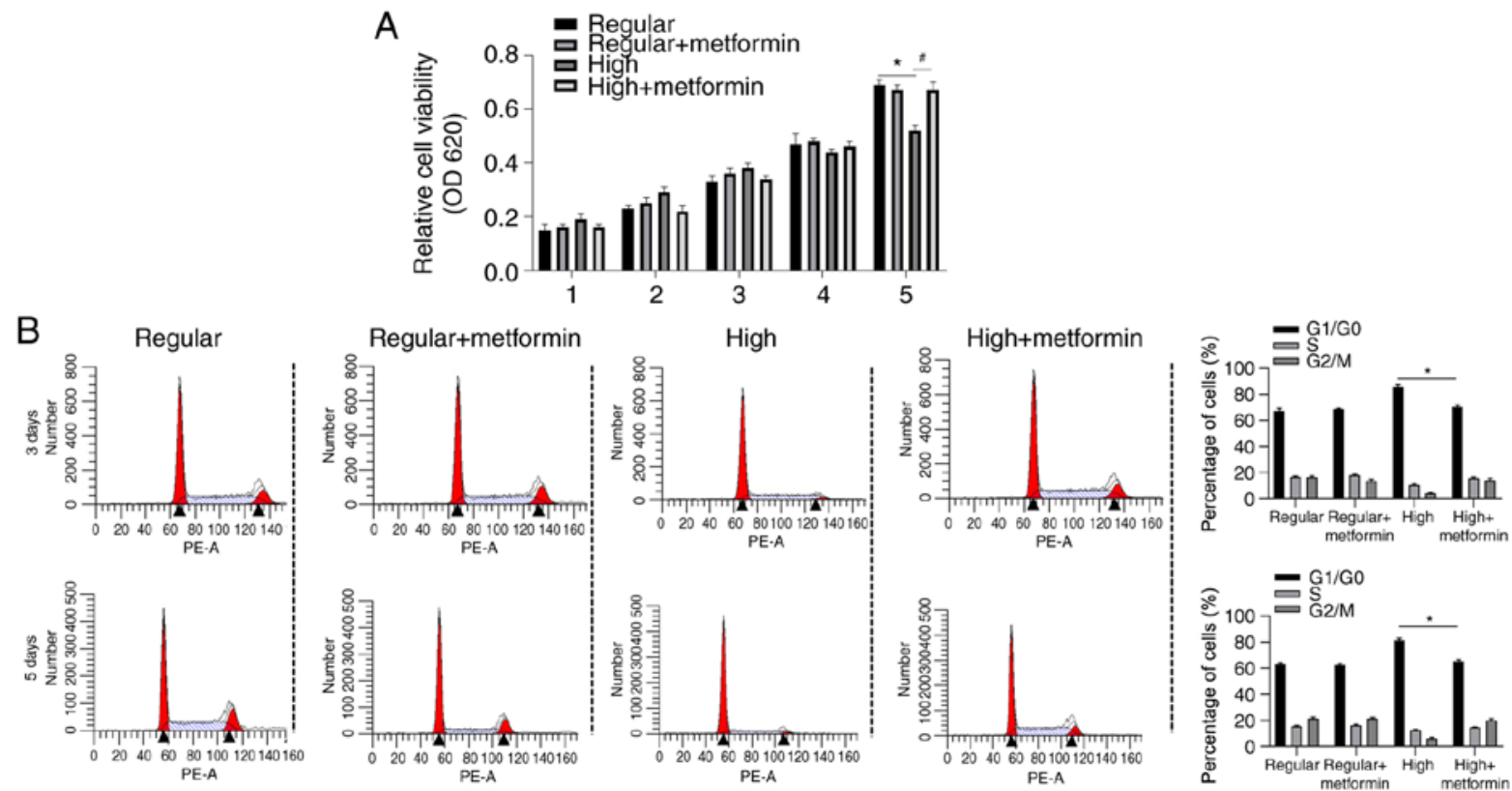

Figure 2. Metformin reverses the inhibitory effect induced by high glucose condition on cell proliferation. (A) Cell viability was assessed via the Cell Counting Kit-8 assay following incubation under regular or high glucose conditions for 1-5 days, respectively. (B) Cell cycle phases were measured via propidium iodide staining, followed by flow cytometric analysis. ${ }^{*} \mathrm{P}<0.05$ vs. regular glucose group; ${ }^{*} \mathrm{P}<0.05$ vs. high glucose group. OD, optical density; PE-A, phycoerythrin label.

A

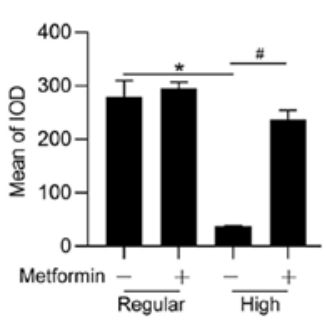

B
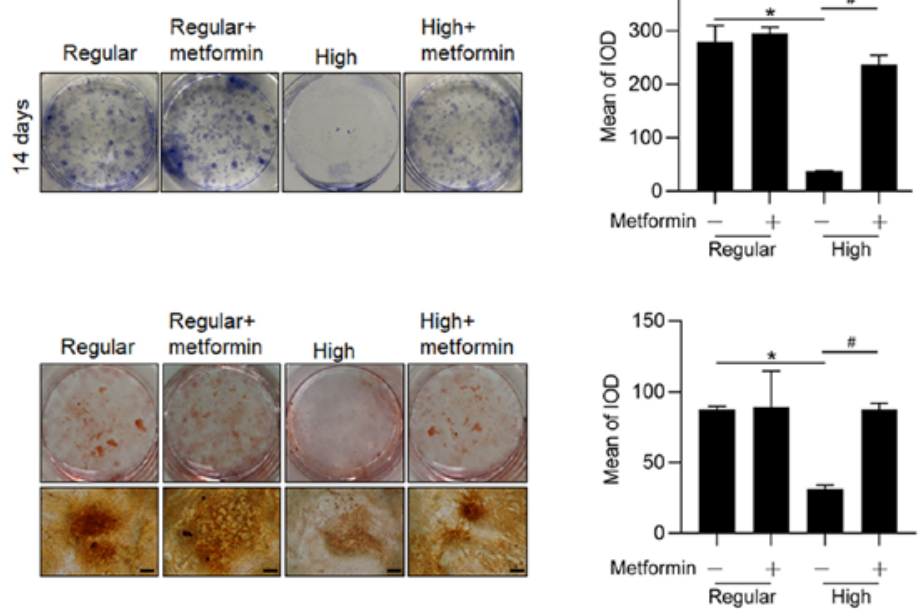

C
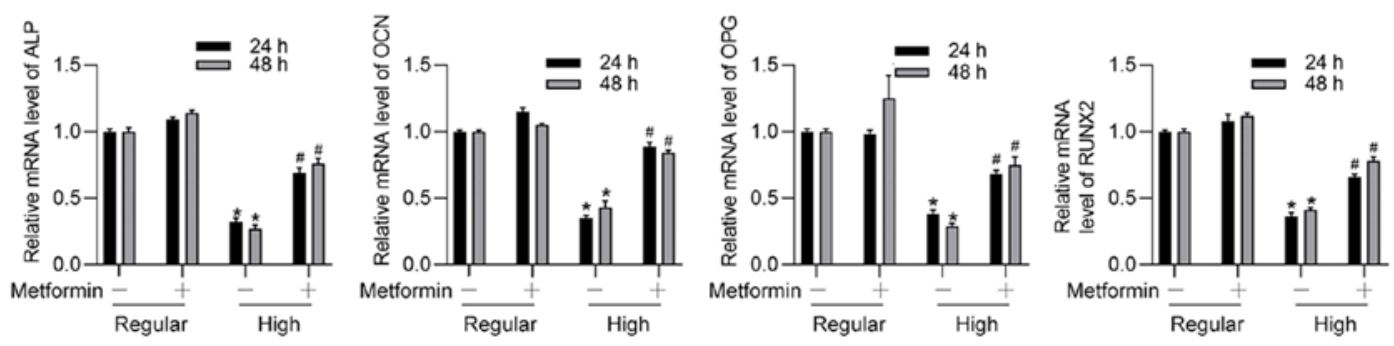

Figure 3. Metformin promotes osteogenic differentiation of BMSCs under high glucose/differentiating condition. (A) ALP and (B) Alizarin Red S staining assays were performed following incubation in differentiating medium under high glucose condition for 14 days, with or without metformin (magnification, $\mathrm{x} 40$; scale bar=50 $\mu \mathrm{m})$. (C) mRNA expression levels of ALP, OCN, OPG and RUNX2, of BMSCs cultured in differentiating medium under regular and high glucose conditions were detected via reverse transcription-quantitative PCR analysis. ${ }^{*} \mathrm{P}<0.05$ vs. regular glucose group; ${ }^{\sharp} \mathrm{P}<0.05$ vs. high glucose group. BMSCs, bone marrow stromal cells; ALP, alkaline phosphatase; OCN, osteocalcin; OPG, osteoprotegerin; RUNX2, runt-related transcription factor 2; IOD, integrated optical density.

were reversed following addition of metformin (Fig. 4A). Notably, metformin failed to affect JC-1 staining under regular glucose condition, indicating its specific role on exerting protective effects against high glucose conditions. The 
A
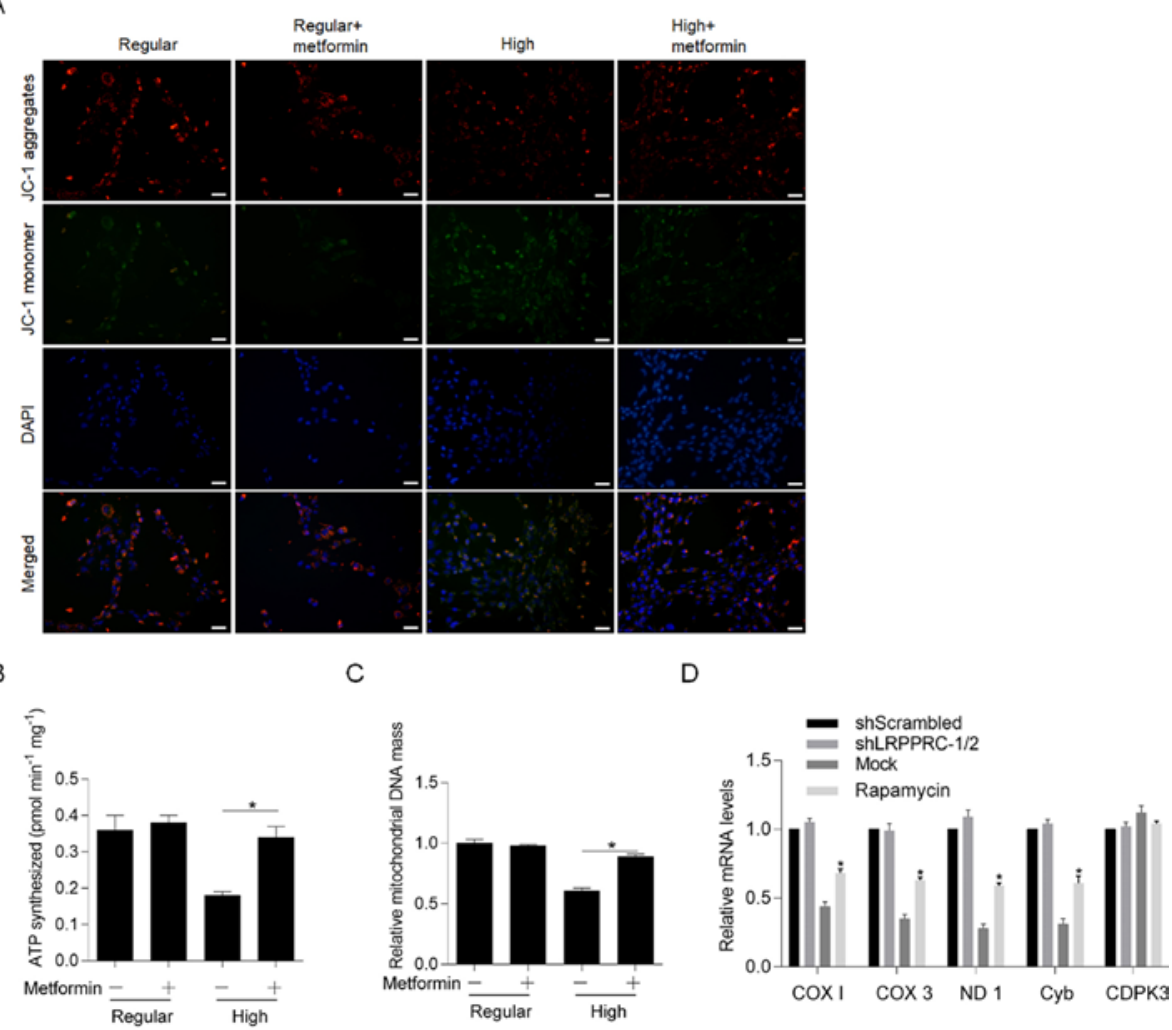

Figure 4. Metformin reverses mitochondrial dysfunction induced by high glucose condition in differentiating medium. (A) JC-1 staining was performed to detect mitochondrial membrane potential (magnification, $\mathrm{x} 40$; scale bar=50 $\mu \mathrm{m}$ ). (B) ATP synthesis was analyzed. (C) Mitochondrial DNA mass was measured via reverse transcription- quantitative PCR. (D) Mitochondrial transcriptional activity was measured by detecting mitochondrial genome-coded genes. ${ }^{*} \mathrm{P}<0.05$ vs. high glucose group.

present study also investigated whether metformin reversed glucose-induced mitochondrial dysfunction by detecting ATP synthesis (Fig. 4B), mitochondrial DNA mass (Fig. 4C) and mitochondrial transcriptional activity (Fig. 4D). As expected, incubation with high glucose for $48 \mathrm{~h}$ at $37^{\circ} \mathrm{C}$ significantly decreased mitochondrial function, whereas co-culture with metformin significantly reversed these effects.

Metformin scavenges ROS induced by high glucose and thus potentially regulates the ROS-AKT-mTOR axis. In order to assess how high glucose affects ROS accumulation in BMSCs cultured in differentiating medium, ROS staining was performed using the DCFH-DA probe kit. The results demonstrated that fluorescence intensity of BMSCs cultured in high glucose condition increased compared to the mock group (Fig. 5A). However, fluorescence intensity significantly decreased following treatment with metformin or antioxidant NAC (Fig. 5A). Considering that the ROS-AKT-mTOR axis plays a critical role in physiological processes of several normal tissues, excluding osteogenesis (19), the protein levels of AKT and mTOR, and their phosphorylated forms were detected. Western blot analysis demonstrated that the protein levels of p-AKT (Thr308) and p-mTOR (Ser2448) significantly decreased under high glucose condition, the effects of which were significantly reversed following co-culture with metformin or NAC (Fig. 5B-D). Collectively, these results suggest that metformin may protect BMSCs from high glucose-induced ROS accumulation, and thus stimulate the ROS-AKT-mTOR axis.

\section{Discussion}

It is well known that diabetes mellitus remarkably increases the risk of osteopathy, such as osteoporosis and fragility fractures, via long-term exposure to high glucose conditions $(1,20)$. Metformin, as the most commonly used antidiabetic drug, has been reported to exert protective effects against bone loss, and thus decreases the risk of diabetic osteopathy (21). Osteogenic cell proliferation and differentiation are key elements affecting bone health (22), and diabetic-associated hyperglycemia negatively affects both proliferation and differentiation of these cells (22). Bone marrow mesenchymal stem cells are responsible for inducing bone damage repair and secreting a large amount of extracellular matrix components to promote fracture healing (23). They are also extensively used for bone engineering and artificial bone repairment (24). Thus, the present study mimicked hyperglycemia by using high glucose conditions to culture BMSCs, and assessed its effects on proliferation and osteogenic differentiation. Specifically, the effects of high glucose levels and metformin on osteogenesis of BMSCs were investigated. Prospective studies will also focus on the role of osteoblasts.

The effects of high glucose exposure on BMSCs osteogenesis under differentiating medium were investigated. The results of the present study were consistent with previous finding, demonstrating that high glucose levels affect osteoblast differentiation (25). The results of the present study demonstrated that high glucose levels inhibited cell 
A
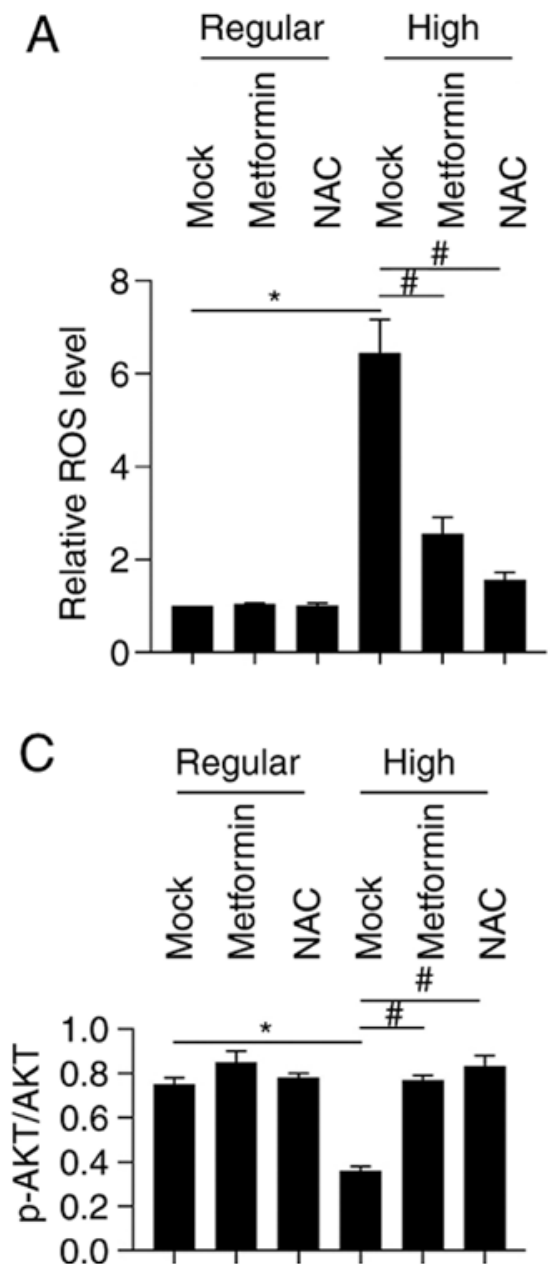

B

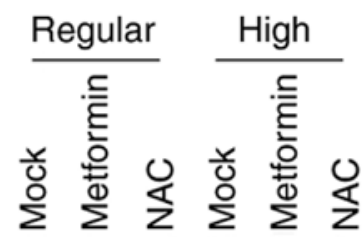

p-AKT

(Thr308)

AKT

p-mTOR

(Ser2448)

mTOR

$\beta$-actin
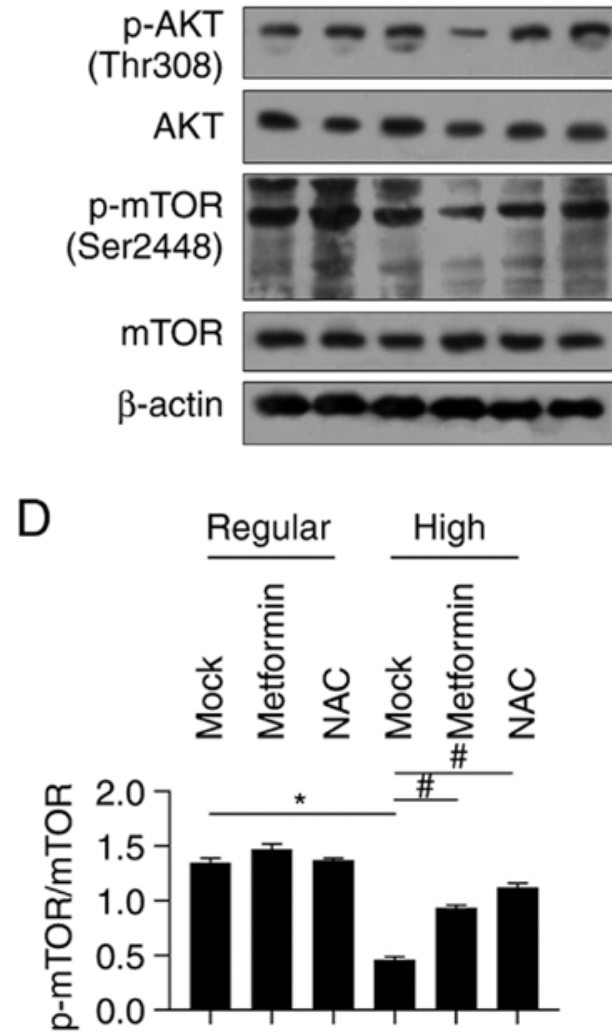

Figure 5. Metformin scavenges ROS accumulation induced by high glucose condition. (A) ROS accumulation was quantitatively measured following incubation for $48 \mathrm{~h}$ at $37^{\circ} \mathrm{C}$. (B) Western blot analysis was performed to detect the protein levels of mTOR, p-mTOR (Ser2448), AKT and p-AKT (Thr308). B-actin was used as the internal control. The ratios of (C) p-AKT/AKT and (D) p-mTOR/mTOR were measured using ImageJ software. ${ }^{*} \mathrm{P}<0.05$ vs. regular glucose group; ${ }^{\sharp} \mathrm{P}<0.05$ vs. high glucose group. ROS, reactive oxygen species; NAC, n-Acetyl Cysteine; mTOR, mammalian target of rapamycin; AKT, serine/threonine kinase 1.

proliferation and differentiation, respectively. Notably, addition of metformin significantly reversed the inhibitory effects induced by high glucose levels on cell proliferation and differentiation. ALP is a plasma membrane-bound glycoprotein, which is extensively expressed in the placenta, intestine, liver, kidneys and bone (26). In the present study, it was used as a marker to assess osteoblast activity. Furthermore, osteogenic-related genes, including OCN, OPG and RUNX2 were also used as osteogenic markers. A previous study reported that metformin promotes osteoblast proliferation and differentiation (14), suggesting that metformin may exert similar effects on high glucose stress in different kinds of cells. Notably, high glucose exposure slightly increased cell viability from day 1 to day 3 , and subsequently decreased cell viability after day 4 . This may because the high glucose levels increased metabolism and increased enzymatic activity in BMSCs. To investigate the promoting effect of high glucose on proliferation, cell cycle distribution was assessed from day 3 , instead of an earlier time point. Notably, no obvious osteogenesis was observed in the growth medium after 14 and 21-day culture, this may due to the low processing rate in growth medium. This indicated the main difference between growth medium and differentiating medium on osteogenesis. A major limitation of the present study is the lack of detection of the absorption of glucose into the cytoplasm following addition of metformin; however, it is speculated that metformin may not affect glucose absorption.

Long-term exposure to high glucose levels leads to metabolic disturbance, causes oxidative stress and induces ROS accumulation (16). A previous study reported that glucose oxidative stress induced by high glucose levels interferes with osteoblast differentiation and mineralization (27). One of the main causes of cell damage is accumulated ROS induced by high glucose levels (27). Mitochondrial biogenesis enhances cellular function, survival and recovery from oxidative-induced cell damage (28). Targeting glucose-induced ROS and protecting mitochondrial DNA from oxidative stress is considered a promising therapeutic strategy for high glucose-induced inhibition of cell proliferation and osteoblastic differentiation (27). Takanche et al reported that gomisin A, a lignan isolated from the hexane of Schisandra chinensis fruit extract, protects osteoblast from high glucose-induced oxidative stress and promotes mitochondrial function (27). The results of the 
present study confirmed that high glucose levels induced ROS accumulation, decreased MMP and caused mitochondrial dysfunction. A reduction in mitochondrial mass or decrease in mitochondrial membrane potential may be two main results of mitochondrial dysfunction. Thus, prospective studies will focus on investigating mitochondrial mass and MMP. Another major limitation of the present study is the lack of confirmation as to how metformin decreases ROS levels, by inhibiting ROS genesis or by scavenging ROS accumulation.

Addition of metformin scavenged ROS induced by high glucose levels, reversed the lack of ATP synthesis and mitochondrial transcriptional activity by high glucose-induced oxidative stress. The ROS-AKT-mTOR axis has been demonstrated to be stimulated under high glucose levels, and affects physiological process, such as proliferation and osteogenesis in osteoblasts (16). The results of the present study demonstrated that metformin decreased ROS levels and activated AKT-mTOR signaling. However, whether metformin regulates the ROS-AKT-mTOR axis directly or indirectly remains unclear. Furthermore, the effect of metformin on osteogenesis without high glucose stimulation remains unknown, and thus is worth investigating in future studies.

The present study is not without limitations. The exact regulatory mechanism of metformin on the ROS-AKT-mTOR axis, and the effect of metformin on bone formation in vivo were not investigated. Thus, prospective studies will focus on investigating the effects of high glucose levels on proliferation and osteogenic differentiation. Taken together, the results of the present study suggest that metformin exerts a protective role on BMSCs from relatively-high glucose-induced oxidative stress.

\section{Acknowledgements}

The authors of the present study would like to thank Ms. Huimin Shi from Sichuan University (Chengdu, China) for helping draft the initial manuscript.

\section{Funding}

No funding was received.

\section{Availability of data and materials}

The datasets used and/or analyzed during the current study are available from the corresponding author on reasonable request.

\section{Authors' contributions}

RZ and XZ contributed to conception and design. RZ, YM and SQ contributed to acquisition of data. RZ and $Z G$ contributed to acquisition, analysis and interpretation of data. ZG and SQ contributed to cell culture. All authors read and approved the final manuscript.

\section{Ethics approval and consent to participate}

All animal experiments were approved by the Ethics Committee of the First Affiliated Hospital of China Medical University.

\section{Patient consent for publication}

Not applicable.

\section{Competing interests}

The authors declare that they have no competing interests.

\section{References}

1. Janghorbani M, Van Dam RM, Willett WC and Hu FB: Systematic review of type 1 and type 2 diabetes mellitus and risk of fracture. Am J Epidemiol 166: 495-505, 2007.

2. Garmendia Madariaga A, Santos Palacios S, Guillén-Grima F and Galofré JC: The incidence and prevalence of thyroid dysfunction in Europe: A meta-analysis. J Clin Endocrinol Metab 99: 923-931, 2014.

3. Dhaliwal R, Cibula D, Ghosh C, Weinstock RS and Moses AM: Bone quality assessment in type 2 diabetes mellitus. Osteoporos Int 25: 1969-1973, 2014.

4. Chen Y, Hu Y, Yang L, Zhou J, Tang Y, Zheng L and Qin P: Runx2 alleviates high glucose-suppressed osteogenic differentiation via PI3K/AKT/GSK3 $\beta / \beta$-catenin pathway. Cell Biol Int 41: 822-832, 2017.

5. Harvey NC, McCloskey EV, Mitchell PJ, Dawson-Hughes B, Pierroz DD, Reginster JY, Rizzoli R, Cooper C and Kanis JA: Mind the (treatment) gap: A global perspective on current and future strategies for prevention of fragility fractures. Osteoporos Int 28: 1507-1529, 2017.

6. Jackuliak P and Payer J: Osteoporosis, fractures, and diabetes. Int J Endocrinol 2014: 820615, 2014

7. Wybier M, Mathieu P, Morvan G, Vuillemin Bodaghi V and Guerini H: Muculoskeletal radiology: ankle and foot in adults. J Radiol 89: 711-724, quis 735-736, 2008 (In French).

8. Rosenbloom AL and Silverstein $\mathrm{JH}$ : Connective tissue and joint disease in diabetes mellitus. Endocrinol Metab Clin North Am 25: 473-483, 1996.

9. Smith DG, Barnes BC, Sands AK, Boyko EJ and Ahroni JH: Prevalence of radiographic foot abnormalities in patients with diabetes. Foot Ankle Int 18: 342-346, 1997.

10. Markowicz Piasecka M, Sikora J, Szydlowska A, Skupien A, Mikiciuk Olasik E and Huttunen KM: Metformin - a future therapy for neurodegenerative diseases: Theme: Drug discovery, development and delivery in Alzheimer's disease guest editor: Davide Brambilla. Pharm Res 34: 2614 2627, 2017.

11. Cortizo AM, Sedlinsky C, McCarthy AD, Blanco A and Schurman L: Osteogenic actions of the anti-diabetic drug metformin on osteoblasts in culture. Eur J Pharmacol 536: 38-46, 2006.

12. Gao Y, Xue J, Li X, Jia Y and Hu J: Metformin regulates osteoblast and adipocyte differentiation of rat mesenchymal stem cells. J Pharm Pharmacol 60: 1695-1700, 2008.

13. Zhen D, Chen Y and Tang X: Metformin reverses the deleterious effects of high glucose on osteoblast function. J Diabetes Complications 24: 334-344, 2010.

14. Shao X, Cao X, Song G, Zhao Y and Shi B: Metformin rescues the MG63 osteoblasts against the effect of high glucose on proliferation. J Diabetes Res 2014: 453940, 2014.

15. Renner K, Seilbeck A, Kauer N, Ugele I, Siska PJ, Brummer C, Bruss C, Decking SM, Fante M, Schmidt A, et al: Combined Metabolic Targeting With Metformin and the NSAIDs Diflunisal and Diclofenac Induces Apoptosis in Acute Myeloid Leukemia Cells. Front Pharmacol 9: 1258, 2018.

16. Wang X, Feng Z, Li J, Chen L and Tang W: High glucose induces autophagy of MC3T3-E1 cells via ROS-AKT-mTOR axis. Mol Cell Endocrinol 429: 62-72, 2016.

17. Park KR, Nam D, Yun HM, Lee SG, Jang HJ, Sethi G, Cho SK and Ahn KS: $\beta$-Caryophyllene oxide inhibits growth and induces apoptosis through the suppression of PI3K/AKT/mTOR/S6K1 pathways and ROS-mediated MAPKs activation. Cancer Lett 312: 178-188, 2011.

18. Livak KJ and Schmittgen TD: Analysis of relative gene expression data using real-time quantitative PCR and the 2(-Delta Delta C(T)) Method. Methods 25: 402-408, 2001.

19. Fiorini C, Cordani M, Gotte G, Picone D and Donadelli M: Onconase induces autophagy sensitizing pancreatic cancer cells to gemcitabine and activates Akt/mTOR pathway in a ROS-dependent manner. Biochim Biophys Acta 1853: 549-560, 2015. 
20. Botushanov NP and Orbetzova MM: Bone mineral density and fracture risk in patients with type 1 and type 2 diabetes mellitus. Folia Med (Plovdiv) 51: 12-17, 2009.

21. Wang C, Li H, Chen SG, He JW, Sheng CJ, Cheng XY, Qu S, Wang KS, Lu ML and Yu YC: The skeletal effects of thiazolidinedione and metformin on insulin-resistant mice. J Bone Miner Metab 30: 630-637, 2012.

22. Sun H, Dai K, Tang T and Zhang X: Regulation of osteoblast differentiation by slit 2 in osteoblastic cells. Cells Tissues Organs 190: 69-80, 2009.

23. Granero-Moltó F, Weis JA, Miga MI, Landis B, Myers TJ, O'Rear L, Longobardi L, Jansen ED, Mortlock DP and Spagnoli A: Regenerative effects of transplanted mesenchymal stem cells in fracture healing. Stem Cells 27: 1887-1898, 2009.

24. Li C, Yang L, Ren X, Lin M, Jiang X, Shen D, Xu T, Ren J, Huang L, Qing W, et al: Groove structure of porous hydroxyapatite scaffolds (HAS) modulates immune environment via regulating macrophages and subsequently enhances osteogenesis. J Biol Inorg Chem 24: 733-745, 2019.
25. Yang J, Ma C and Zhang M: High glucose inhibits osteogenic differentiation and proliferation of MC3T3 E1 cells by regulating P2X7. Mol Med Rep 20: 5084-5090, 2019.

26. Sharma U, Pal D and Prasad R: Alkaline phosphatase: An overview. Indian J Clin Biochem 29: 269-278, 2014.

27. Takanche JS, Kim JE, Han SH and Yi HK: Effect of gomisin A on osteoblast differentiation in high glucose-mediated oxidative stress. Phytomedicine 66: 153107, 2020.

28. Liu J, Shen W, Zhao B, Wang Y, Wertz K, Weber P and Zhang P: Targeting mitochondrial biogenesis for preventing and treating insulin resistance in diabetes and obesity: Hope from natural mitochondrial nutrients. Adv Drug Deliv Rev 61: 1343-1352, 2009.

(c) (i) () $($ This work is licensed under a Creative Commons

Cy No No Attribution-NonCommercial-NoDerivatives 4.0 International (CC BY-NC-ND 4.0) License. 